\title{
Comprehensive follow up care reduced life threatening illnesses without increasing costs in high risk, inner city infants
}

\author{
Broyles RS, Tyson JE, Heyne ET, et al. Comprehensive follow-up care and life-threatening illnesses among high-risk infants. \\ A randomized controlled trial.JAMA 2000 Oct 25;284:2070-6.

\section{QUESTIONS: Does access to comprehensive follow up care reduce life threatening illnesses among high risk, inner city infants? Is it cost effective?}

\section{Design}

Randomised (allocation concealed), blinded (outcome assessors) controlled trial with follow up to age 1 year.

\section{Setting}

A follow up clinic in a private children's hospital in Dallas county, Texas, USA.

\section{Patients}

887 infants who either weighed $<1000 \mathrm{~g}$ at birth or weighed between 1001 and $1500 \mathrm{~g}$ and received mechanical ventilation in the first 48 hours after birth. 783 infants (88\%) were included in the primary analysis (mean maternal age 23 y; $54 \%$ black, $31 \%$ Hispanic). At 1 year follow up, 37 infants had unknown outcomes.

\section{Intervention}

446 infants were allocated to comprehensive follow up care and 441 were allocated to routine care. Both groups were cared for by the same highly experienced clinic staff (2 paediatric nurse practitioners [NPs] and a physician's assistant [PA], each supervised by both a paediatrician and neonatologist trained in follow up care). Comprehensive care was provided 5 days per week and included all components of routine care as well as care for acute illnesses. The NP or PA was available by telephone or pager at all hours for acute problems, and contacted the physician supervisor or emergency department (ED) staff if infants required immediate care outside of clinic hours. Routine care was available 2 mornings per week and included standard well baby care and care for chronic illnesses. Mothers were taught the signs of acute illness requiring prompt evaluation and told to seek care in neighbourhood clinics on weekdays or the ED at other times.

Sources of funding: Agency for Healthcare Research and Quality: North Texas Chapter of the National Foundation March of Dimes.

For correspondence: of Texas-Houston Medical School, 6431 Fannin Street, MSB 3.228, Houston, $T X$ 77030-1503, USA Fax +17135000519 . DrJ E Tyson, University

\section{Main outcome measures}

Main outcomes were life threatening illness (illness resulting in death or admission to the intensive care unit [ICU]), admission to ICU, death, and costs (inpatient and outpatient).

\section{Main results}

Analysis was by intention to treat. Between nursery discharge and 1 year of age, fewer infants who received comprehensive care had life threatening illnesses and fewer received paediatric intensive care (table). The

Comprehensive follow up care $v$ routine care for high risk, inner city infants*

\begin{tabular}{lllll} 
Outcomes at age 1 year & $\begin{array}{l}\text { Comprehensive } \\
\text { care }\end{array}$ & $\begin{array}{l}\text { Routine } \\
\text { care }\end{array}$ & RRR (95\% Cl) & NNT (Cl) \\
\hline Life threatening illness & $8 \%$ & $16 \%$ & $48 \%(22$ to 65$)$ & 14 (9 to 33$)$ \\
\hline Admission to intensive care & $6 \%$ & $13 \%$ & $57 \%(31$ to 73$)$ & 14 (9 to 29$)$ \\
\hline
\end{tabular}

*Abbreviations defined in glossary; RRR, NNT, and $\mathrm{Cl}$ calculated from data in article. groups did not differ for deaths $(11 v 13, \mathrm{p}=0.68)$ or for the estimated mean cost per infant (US\$6265 v\$9913).

\section{Conclusion}

Among high risk, inner city infants, comprehensive follow up care reduced life threatening illnesses and admission to intensive care in the first year of life, without increasing overall costs.

\section{COMMENTARY}

Little is known about the outcomes of neonatal follow up programmes for infants of families of lower socioeconomic status. Even less documentation is available about programmes for very low birth weight infants. This well designed and executed study by Broyles $e t a l$ is one of the few that provides cost and quality outcome data for this vulnerable population. Brooten et al assessed an early discharge programme by advanced practice nurses for high risk infants and found impressive cost savings, ${ }^{1}$ as did Broyles $e t$ al. Although the study by Broyles focused on a somewhat different intervention, the interventions in both studies provided additional care after discharge to a similar population.

Strengths of the study include its large sample size, request for parental consent before hospital discharge to reduce loss to follow up, random allocation of infants to groups with stratification by birth weight to optimise similarity of groups, blinded outcome assessment, and follow up of $88 \%$ of the sample to 1 year of age.

More than $80 \%$ of the mothers in this study were black or Hispanic, and their mean education was $<11$ years. The results may not be generalisable therefore to populations with different racial or educational backgrounds. The health professionals who provided the follow up care to both groups were highly experienced NPs and a PA. Replication of this intervention with less experienced or different types of care providers might yield dissimilar results.

The results are relevant to nurses who work in neonatal follow up or developmental clinics, paediatric nurses and NPs, neonatologists, paediatricians, and administrators of developmental follow up programmes. Inner city high risk infants are particularly vulnerable because their care is often limited and fragmented and minor acute illnesses or complications can quickly become life threatening and costly. At a time when funding for traditional follow up clinics is declining, this study provides strong outcome and cost based rationale for implementation of comprehensive follow up care for vulnerable populations.

Rosanne C Harrigan, APRN-Rx, EdD, FAAN Dean, Professor, and Frances A Matsuda Chair in

Women's Health

School of Nursing and Dental Hygiene University of Hawaii at Manoa Honolulu, Hawaii, USA

1 Brooten D, Kumar S, Brown LP, et al. A randomised clinical trial of early hospital discharge and home follow-up of verylow-birth-weight infants. N Engl J Med 1986;315:934-9. 